\title{
A Helically Symmetric Stellarator (HSX)
}

\author{
A. F. Almagri, D. T. Anderson, F. S. B. Anderson, P. H. Probert, J. L. Shohet, and J. N. Talmadge
}

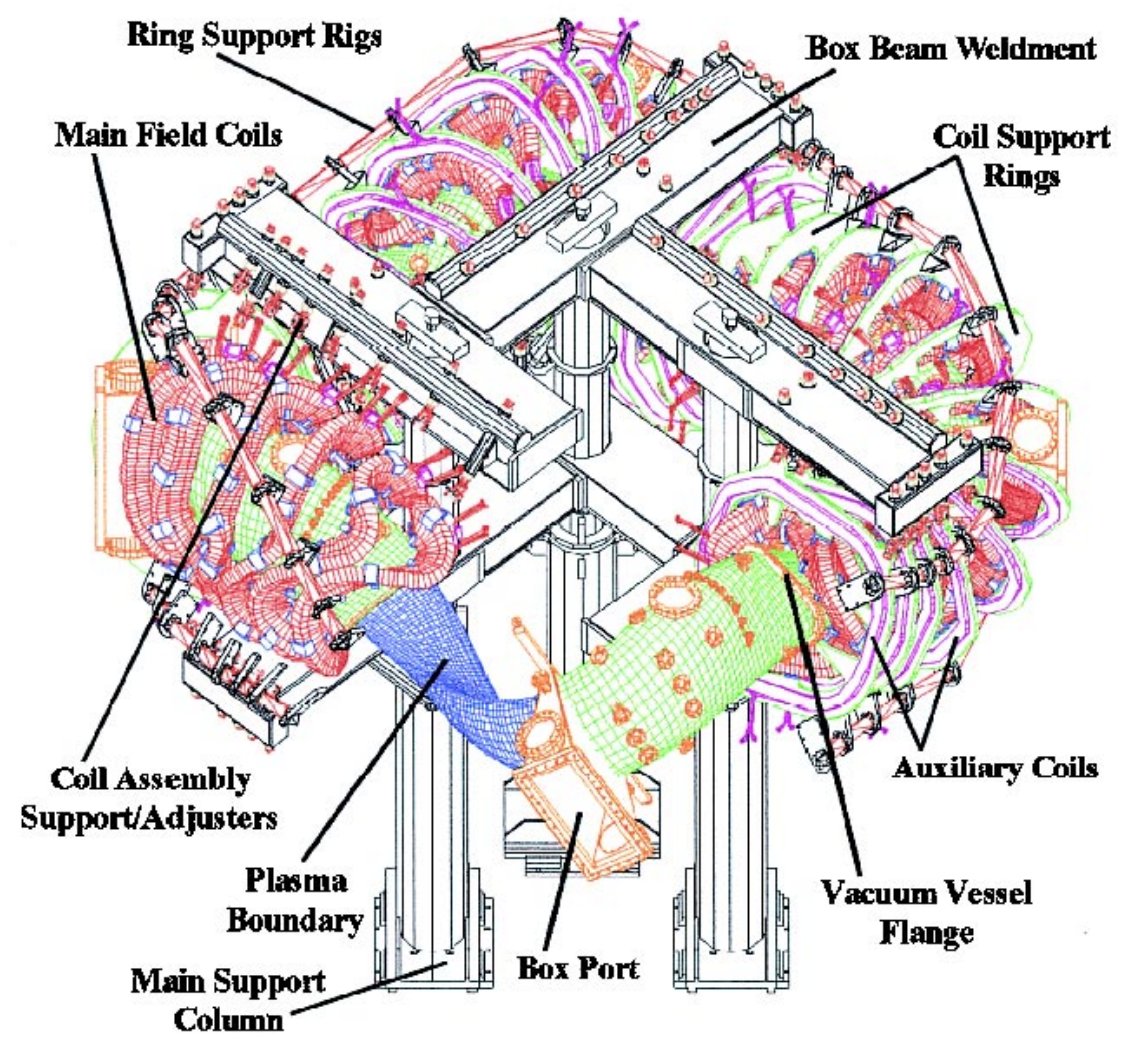

Fig. 1. An isometric view of the HSX stellarator device showing the key features of the device beginning with the plasma boundary, the vacuum chamber, and the main and auxiliary magnet coils along with the support structure.

\begin{abstract}
The Helically Symmetric Experiment (HSX) is a quasi-helically symmetric (QHS) stellarator currently under construction at the Torsatron/Stellarator Laboratory of the University of Wisconsin-Madison. This device is unique in its magnetic design in that the magnetic field spectrum possesses only a single dominant (helical) component. This design avoids the large direct orbit losses associated with conventional stellarators. The restoration of an ignorable coordinate in the confining magnetic field makes this device analogous to an axisymmetric $q=1 / 3$ tokamak with respect to neoclassical confinement.
\end{abstract}

A XISYMMETRIC toroidal fusion research devices (e.g., tokamaks) possess magnetic field symmetries which ensure good single-particle confinement and are conceptually simple in magnet coil design. However, the pulsed nature of

Manuscript received July 1, 1998; revised August 28, 1998. This work was supported by the U.S. Department of Energy under Grant DE-FG0293ER54222.

The authors are with Torsatron/Stellarator Laboratory, University of Wisconsin-Madison, Madison, WI 53706 USA (e-mail: shohet@engr. wisc.edu).

Publisher Item Identifier S 0093-3813(99)02419-4. inductive current drive, the large source of free energy in the plasma current which can drive instabilities (disruptions), the requirements for auxiliary and divertor magnet coil sets for plasma shaping, and for stability and position control, force increased engineering costs and complexities for such devices. Stellarators, however, have long been considered attractive fusion reactor candidates from the point of view of no required ohmic current, permitting true steady-state operation. The magnet coil sets for such devices, although complicated, are no more so than present-day tokamak devices.

In conventional axisymmetric toroidal systems such as tokamaks, two magnetic field components produce the confining fields. They are 1) a conventional toroidal magnetic field and 2) a "poloidal" magnetic field produced by an ohmic current induced in the toroidal direction. The axis of symmetry therefore, is the toroidal direction. Classical stellarators, however, use helical coils to produce both the poloidal and toroidal magnetic fields which have magnetic field variations in the toroidal direction and therefore a breaking of the symmetry as compared to a tokamak. This gives rise to significant deviation of the particle drift surfaces off the magnetic flux surfaces 
and transport that increases with increasing temperature in the regime where fusion is likely to take place.

To produce a stellarator configuration with an axis of symmetry, the magnet coils were specially designed as follows. First, the desired helically symmetric magnetic surfaces were specified. Then, the magnet coils were designed to produce these fields. In so doing, symmetry was confirmed in the stellarator configuration. Fig. 1 shows an isometric view of the Helically Symmetric Experiment (HSX) stellarator device. There are a total of 48 individual main coils with six different shapes of these coils. The magnet arrangement repeats itself four times in going around the torus, (four field periods) with the six magnet coils being rotated about the mid plane of each coil to complete a $1 / 4$ toroidal section. Each coil is supported with a stainless steel ring that is anchored to a central support frame through adjusters that are turnbuckle-like connections. On each support ring is an auxiliary coil-planar, but noncircular - that is used to break the helical symmetry in order to compare the transport properties of a QHS stellarator to one without helical symmetry.

The vacuum chamber, which is mounted inside the magnet coil set, is shown in Fig. 2.

The vacuum chamber closely follows the shape of the last closed magnetic surface, stepped out to $3 \mathrm{~cm}$ to provide clearance between the vessel and the plasma. Vessel fabrication was performed by explosively forming stainless steel plates that were placed into an accurately CNC-milled mold which conforms to the exterior vessel shape to within $0.025 \mathrm{~cm}$. The vessel is formed in eight sections which are joined at the "corners" using a large box port. In between the box ports is a demountable flange to provide interior access to the vessel.

The dimensions and other technical data for HSX are as follows shown in Table I.

The HSX device has been designed with a clear set of primary physics goals: 1) demonstrate the feasibility of construction of a QHS device, 2) examine single particle confinement with regard to magnetic field symmetry breaking, 3) compare density and temperature profiles in this helically symmetric

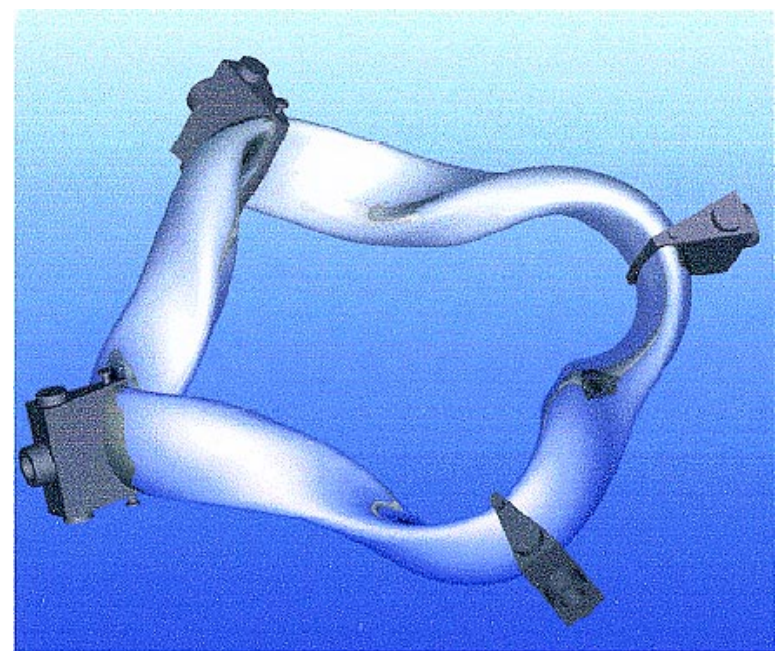

Fig. 2. The stellarator vacuum chamber. The four field-period shape is readily apparent.

TABLE I

PhysicAl PARAMETERS OF THE HSX

\begin{tabular}{l|l|l|l}
\hline Major Radius & $1.2 \mathrm{~m}$ & Avg. coil radius & $0.3 \mathrm{~m}$ \\
\hline Plasma Radius & $0.15 \mathrm{~m}$ & Turns per coil & 14 \\
\hline Plasma Volume & 0.44 cubic meters & Coil current & $13.4 \mathrm{kA}$ \\
\hline ECH Power & $200 \mathrm{~kW}$ & Maximum Field & 1.37 Tesla \\
\hline ECH Frequency & $28 \mathrm{GHz}$ & Pulse Length & $<0.2$ seconds \\
\hline-
\end{tabular}

system to those for axisymmetric tokamaks and conventional stellarators, 4) examine electric fields and plasma rotation with edge biasing in relation to $\mathrm{L}-\mathrm{H}$ transitions in symmetric versus nonsymmetric stellarator systems, 5) investigate QHS effects on low collisionality (high-temperature) regime electron confinement, and 6) examine how greatly-reduced neoclassical electron thermal conductivity compares to the experimentally measured electron thermal conductivity. 